\title{
BMJ Open Gabapentinoids for chronic low back pain: a protocol for systematic review and meta-analysis of randomised controlled trials
}

\author{
Harsha Shanthanna, ${ }^{1}$ Ian Gilron, ${ }^{2}$ Lehana Thabane, ${ }^{1,3}$ Philip J Devereaux, ${ }^{3,4}$ \\ Mohit Bhandari, ${ }^{3,5}$ Rizq AIAmri, ${ }^{1}$ Manikandan Rajarathinam, ${ }^{1}$ Sriganesh Kamath ${ }^{1,6}$
}

To cite: Shanthanna $\mathrm{H}$, Gilron I, Thabane L, et al. Gabapentinoids for chronic low back pain: a protocol for systematic review and metaanalysis of randomised controlled trials. BMJ Open 2016:6:e013200 doi:10.1136/bmjopen-2016013200

- Prepublication history and additional material is available. To view please visit the journal (http://dx.doi.org/ 10.1136/bmjopen-2016013200)

Received 28 June 2016 Revised 29 September 2016 Accepted 21 October 2016

CrossMark

For numbered affiliations see end of article.

Correspondence to Dr Harsha Shanthanna; harshamd@gmail.com

\section{ABSTRACT}

Introduction: Chronic low back pain (CLBP) is a common condition and causes significant pain, distress and disability across the world. It is multifactorial in aetiology and is challenging to manage. Although the underlying mechanism of pain is predominantly non-specific, many argue that there is a substantial neuropathic pain element. Neuropathic pain is more severe, with significant disability. Gabapentinoids, including gabapentin and pregabalin, have proven efficacy in some neuropathic pain conditions. Despite no clear evidence, a substantial population of patients with CLBP are treated with gabapentinoids.

Objectives: We aim to assess whether the use of gabapentinoids is effective and safe in the treatment of predominant CLBP, by conducting a systematic review and meta-analysis of randomised control trials (RCTs). Methodology: We will search the databases of MEDLINE, EMBASE and Cochrane for RCTs published in English language and have used gabapentinoids for the treatment of CLBP. Study selection and data extraction will be performed independently by paired reviewers using structured electronic forms, piloted between pairs of reviewers. The review outcomes will be guided by Initiative on Methods, Measurement and Pain Assessment in Clinical Trials guidelines, with pain relief as the primary outcome. We propose to carry out meta-analysis if there are three or more studies in a particular outcome domain, using a random effects model. Pooled outcomes will be reported as weighted mean differences or standardised mean differences and risk ratios with their corresponding $95 \% \mathrm{Cls}$, for continuous outcomes and dichotomous outcomes, respectively. Rating of quality of evidence will be reported using GRADE summary of findings table.

Discussion: The proposed systematic review will be able to provide valuable evidence to help decisionmaking in the use of gabapentinoids for the treatment of CLBP. This will help advance patient care and potentially highlight limitations in existing evidence to direct future research.

Ethics and dissemination: Being a systematic review, this study would not necessitate ethical review and approval. We plan to report and publish our study

\section{Strengths and limitations of this study}

- There are no existing reviews on the use of gabapentinoids for predominant, chronic low back pain (CLBP).

- Our review methodology incorporates a detailed risk of bias assessment, including elements that have been proposed specifically for chronic pain trials by well-known Cochrane pain researchers.

- Our review team consists of experts in the field of analgesia drug trials and also experienced research methodologists.

- Our review involves select population of predominant CLBP and hence may limit its applicability to patients with leg and back pain or predominant leg pain.

- Our review, and hence its results, would be limited by the number and quality of randomised controlled studies in this area.

- Owing to the variability involved in the study population, and also in the way of outcome measurements, our results may carry substantia heterogeneity.

findings in a high impact medical journal, with online access.

Trial registration number: CRD42016034040.

\section{BACKGROUND}

\section{Burden of chronic low back pain}

Chronic low back pain (CLBP) is very common. It is typically considered to be pain felt in the area of the low back and lasting at least 12 weeks or more in duration. ${ }^{12}$ Exact estimates of the prevalence of CLBP are difficult to establish because of the variability in the questions and criteria used in epidemiological studies. ${ }^{3}$ Many studies looking at the burden of CLBP have included population with acute $(<12$ weeks) low back pain 
(LBP). ${ }^{3}$ The life time prevalence of LBP-not necessarily chronic, varies between $51 \%$ and $80 \% .^{1}$ A majority of these episodes are self-limiting. When CLBP alone is considered, it is estimated to be around 5.9-18.1\%. ${ }^{14}$ CLBP causes significant pain, suffering, impairment of daily activities, and decreased quality of life. ${ }^{4}$ Among chronic conditions CLBP has been noted to be the leading cause of years lived with disability. ${ }^{5}$

\section{Aetiological considerations of CLBP}

Axial CLBP is multifactorial and in many patients diffuse and non-specific. ${ }^{6}$ There are several musculoskeletal structures within and around the neuroaxial canal capable of structural damage leading to physiological pain. ${ }^{17}$ On an aetiological and therapeutic perspective, CLBP with sciatica or neurogenic claudication needs to be separated from predominant or isolated CLBP. ${ }^{8}$ Nearly $85 \%$ of isolated CLBP lacks a clear pathoanatomical diagnosis. ${ }^{9}$ On the basis of the underlying nature of pain mechanism, chronic pain conditions could be considered to be either 'neuropathic pain' (NP), or 'non-neuropathic pain' (NNP), ${ }^{10}{ }^{11}$ also referred to as nociceptive. ${ }^{11}$ Central sensitisation (CS) is another category that is supposed to be distinct, but can have overlapping features, within the mechanism-based classification. ${ }^{12} 13$ It is proposed that CS type of pain may be involved in a large number of CLBP patients. ${ }^{14-16}$ In general, identifying a condition as NP in nature carries important implications for diagnosis and management. It has been suggested that NP conditions are more painful, are associated with greater levels of physical and psychological dysfunction and are challenging to treat. ${ }^{17} 18$ Within the CLBP patients, the diagnosis of NP is a challenge. Most epidemiological studies depend on the screening questionnaires presently available in patients of predominant CLBP ${ }^{19}{ }^{20}$ On the basis of studies using screening questionnaires, a recent review suggested a median rate of $41 \%$ with a range of $17-55 \%$ of primary NP. ${ }^{10}$ Others have reported a much lower rate of $4 \% .{ }^{21}$ Data from a US health insurance database showed that the claims for back and neck pain with neuropathic involvement is the most frequent neuropathic disorder. ${ }^{22}$

\section{Treatment considerations in CLBP}

CLBP requires a multidisciplinary approach, ${ }^{3} 7$ and in practice, medications remain an important modality of treatment. ${ }^{23} \mathrm{Up}$ to $80 \%$ of patients in the US are prescribed one or more drugs for LBP in their first visit. ${ }^{3}$ Among the antiepileptics, pregabalin (PG) and gabapentin (GP) are commonly used for many NP conditions. ${ }^{24-26}$ These two medications, grouped together as gabapentinoids, act by $\alpha-2$ delta2 subunit of presynaptic voltage-dependent calcium channels, there by modulating pathologically enhanced neurotransmission in the primary afferent neurons. ${ }^{27} 28$ The use of both medications necessitates slow initiation and titration of dosage and a significant increase in overall treatment costs. ${ }^{29}$ The treatment with gabepentinoids can also be associated with side effects. The side effects common to these medications commonly include sedation, dizziness, peripheral oedema, dry mouth, drowsiness, fatigue, nausea and weight gain. ${ }^{30} 31$

\section{Limitations of existing evidence for the treatment of CLBP}

Analgesic effectiveness of most treatments on nonspecific CLBP is considered to be small. ${ }^{6}$ Although there have been several systematic reviews on the effectiveness of medications for the treatment of CLBP, none of the reviews have specifically reviewed the evidence for the effectiveness and safety of the use of gabapentinoids. White $e t a l^{23}$ were able to assess the effects of nonsteroidal anti-inflammatory drugs (NSAIDs), opioids and antidepressants on CLBP. They observed that NSAIDs were helpful, but antidepressants were no more helpful than placebo with respect to pain, functional status or depression, ${ }^{23}$ although a previous meta-analysis by Salerno et $a \hat{l}^{2}$ had observed that antidepressants were better than placebo. In a recent review, Chou and Huffman reviewed medications for acute and chronic LBP conditions in a review of the evidence for American Pain Society/American College of Physicians Clinical Practice Guideline development. Among medications for CLBP, they found small to moderate benefit with tricyclic antidepressants, and GP in patients with radiculopathy associated with CLBP ${ }^{33}$ This was based on three small trials of GP. They did not identify trials with predominant axial CLBP. The review by Morlion identified two studies for PG and one study for GP. They did not perform a meta-analysis and observed that $P G$ is only effective in a combination therapy. ${ }^{27}$ More recently, Romano $e t a b^{34}$ performed a systematic review of antineuropathic and antinociceptive drugs in patients with CLBP. They also observed that PG combined with celecoxib or opioids was more effective than either monotherapy, based on two small studies. Overall the benefits of treating patients with predominant CLBP by either GP or PG are not clear. We aim to perform a systematic review and meta-analysis to look at the evidence to support the use of gabapentinoids in the treatment of CLBP.

\section{OBJECTIVES}

Primary objectives of this systematic review are: (1) to assess the effectiveness of PG and gabapentin (GB) for pain relief in patients with predominant CLBP; and (2) to assess the safety of using PG and GB in patients with predominant CLBP.

The secondary objectives of this review are as follows: (1) assessing the effects of PG and GB on the Initiative on Methods, Measurement and Pain Assessment in Clinical Trials (IMMPACT) outcomes; ${ }^{35}$ these outcomes include physical functioning, emotional functioning, participant ratings of global improvement and satisfaction with treatment, and participant disposition and (2) 
to assess whether PG and GB selectively improve pain relief in patients with predominant neuropathic CLBP.

\section{METHODS AND ANALYSIS}

Our review protocol has been registered with PROSPERO with the registration number CRD42016034040. This protocol has been prepared for publication according to PRISMA-P guidelines. ${ }^{36}$

\section{Eligibility criteria}

Participants

We will include studies with adult ( $\geq 18$ years of age) patients with CLBP of 3 months or more, with or without lower limb pain. Studies with patients of back and leg/radicular pain will only be included if the population consisted of predominant CLBP, rather than leg/ radicular pain. If a trial involves a mix of CLBP and other chronic pain patients, we will include the study only if they report outcomes separately for our study population of interest, or if at least $90 \%$ of the trial patients are $>18$ years with predominant CLBP.

\section{Studies}

Randomised controlled trials (RCTs) published in English will be eligible for our review.

\section{Interventions}

Eligible studies must randomise patients to receive ' $\mathrm{PG}$ ' or 'GB', either 'alone' or 'in combination with other treatment', and compare it with any active or inactive treatments. We will separately consider the comparisons of active and inactive treatments for pooling.

\section{Information sources}

We will search the electronic databases of EMBASE, MEDLINE and the Cochrane Central Registry of Controlled Trials (CENTRAL), from their inception until 26 January 2016. Our search will be limited to reports published in English. Further, we will search the WHO clinical trial registry (http://apps.who.int/ trialsearch/Default.aspx), and clinical trial registry (https://clinicaltrials.gov/), to look for any registered studies, which fulfil our eligibility criteria and crosscheck for their published results. Unpublished, but completed study results will be requested from the authors or investigators. To further ensure comprehensiveness, we will review the bibliographies of recent reviews and selected studies.

\section{Search strategy}

The search will be performed using a sensitive strategy, in consultation with an experienced librarian, for each specific database. The search terms will include terms referring to study population of low back pain, and terms referring to study interventions-GB, PG and anticonvulsants (see online supplementary appendix 1). We will limit our search to English language.
Non-randomised trials would be excluded during the study selection process.

\section{Study screening and selection}

Study selection will be performed in two stages, with paired reviewers screening studies independently and in duplicate. The first level will be performed on titles and available abstracts, and full text screening will be performed on citations felt potentially eligible by either reviewer. To ensure consistency, reviewers will perform a calibration exercise, before beginning with screening. Reviewers will be asked to resolve disagreement by consensus or, if a discrepancy remains, through discussion with an arbitrator (HS). A quadratic kappa statistic on the full article final decisions will be calculated as a measure of interobserver agreement, independent of chance regarding study eligibility and interpreted as almost perfect agreement (0.81-0.99); substantial agreement (0.61-0.80); moderate agreement $(0.41-0.60)$; fair agreement $(0.21-0.40)$; slight agreement $(0.01-0.20) ;<0$ as less than chance agreement. ${ }^{37}$

\section{Data management}

\section{Data collection process}

Paired reviewers will extract the data independently and in duplicate, using electronic data extraction forms. The forms will be specifically adapted to the present review and will be piloted between the paired reviewers for consistency and accuracy. To assist with the data extraction, an instruction manual will be provided along with each relevant form.

\section{Data items}

Extracted data will include study characteristics, risk of bias items, demographic information, participant flow through the study and outcomes on continuous and binary measures captured on six core domains as recommended by the IMMPACT statement guidelines. ${ }^{35}$

\section{Outcomes and prioritisation}

We will consider pain relief and safety as our primary outcomes and other outcomes (as guided by IMMPACT) as secondary outcomes. We will also prioritise the use of intention to treat analysis (ITT). We will only pool data across trials if there are three or more studies contributing to an outcome domain. Since PG or GB can be used alone or in combination, we will consider pooling studies using PG or GB, either alone or in combination separately. For the primary outcome of pain relief, we will extract continuous outcomes and dichotomous outcomes (success/failure) reported in each study, at various time points. For pooling across studies, we will use the most common outcome type reported. If we consider pooling using the continuous outcomes, we will convert all into a common 0-10 Numerical Rating Scale (NRS), as it is commonly used, and easy to interpret. ${ }^{35}$ We will capture baseline and end scores and change scores. We will prioritise change scores, if reported, for 
the analysis. We will consider the pain relief outcomes reported at the most common time point or the longest follow-up time point for pooling. Safety will be assessed by comparing the risk of serious adverse events causing death, hospitalisation or treatment or study withdrawal. Secondary outcomes will include the comparisons of improvement in physical functioning, emotional functioning and participant ratings of global improvement and satisfaction.

\section{Data synthesis and analysis of outcomes}

Extracted data will be compiled in Microsoft EXCEL for analysis. Risk of bias will be assessed using Cochrane modified risk of bias tool. Included study characteristics will be noted in a table. For the primary analysis, we will use a complete case analysis with ITT. Analysis and synthesis will be carried out using Review Manager (RevMan) (Computer program), V.5.3, Copenhagen: The Nordic Cochrane Centre, The Cochrane Collaboration, 2014; and Microsoft Excel 2011 (Mac version). Using random effects model for pooling, we will calculate either the risk ratio (to be interpreted as the risk of having success) for dichotomous outcomes and weighted mean difference (WMD) or standardised mean differences (SMD) for continuous outcomes, as appropriate. We will consider the inclusion of crossover studies for analysis if the study includes a reasonable washout period to deal with carryover effects, and in which the order of receiving treatments was randomised. For pooling, we will consider results reported from paired test. If not provided, we will consider results of unpaired test (similar to a parallel group trial), and noting that it is conservative, as it will receive less weight. If there is a strong possibility of carryover effect, or if the final results are poorly reported, or if there is a significant drop out rate $(>20 \%)$, we will include the results from the first period only. ${ }^{38}$

\section{Risk of bias assessment and identification}

Risk of bias within the included studies will be assessed using the Cochrane risk of bias tool based on the components of random sequence generation; allocation concealment; blinding of participants; blinding of outcome assessment; incomplete outcome data and selective outcome reporting. For our review, the possibility of selective outcome reporting will be when the outcomes are described in the methods section but not identified or reported in the results section of the same study report. ${ }^{39}$ Among trials of chronic pain treatment, there is a potential for bias with outcome assessment time and the threshold used to establish the success of treatment based on improvement in pain relief. We will consider outcome assessment $<12$ weeks, and $<30 \%$ improvement in pain relief as indicators of potential bias, as suggested by Moore et al. ${ }^{40}$ We will use a modified Cochrane risk-of-bias instrument, with response options of 'definitely yes', 'probably yes', 'probably no' and 'definitely no'" We will assign trials in the 'definitely yes' and 'probably yes' categories a high risk of bias and those in the 'probably no' and 'definitely no' categories a low risk of bias. Any disagreement on the risk of bias item scoring will be noted and arbitrated by the primary investigator (HS). For crossover trials, we will also identify the potential bias resulting from carryover effect, order of randomisation and analysis method. ${ }^{38}$

\section{Assessment of heterogeneity}

Statistical heterogeneity will be calculated using Cochrane's $Q$ test, with a threshold of $p$ value at 0.10 , and the percentage variability in individual effect estimates will be described by $\mathrm{I}^{2}$ statistic. We will consider the $\mathrm{I}^{2}$ threshold as $0-40 \%$ : might not be important; 30 60\%: may represent moderate heterogeneity; 50-90\%: may represent substantial heterogeneity and $75-100 \%$ : considerable heterogeneity, as suggested in the Cochrane handbook. ${ }^{38}$ To explain heterogeneity of $>40 \%$, we will consider the following a priori hypotheses: differences in population, duration of CLBP, dosages of intervention, treatment duration, treatment combinations and outcome measurement standards.

\section{Subgroup analysis}

In studies that have separately reported pain relief in patients who were screened for the presence of NP, we will perform a subgroup analysis to look for the effect of our study interventions (GB or $\mathrm{PG}$ ) on pain relief. These patients will be considered to be NP if they are screened for the presence of leg pain along with CLBP, or NP is identified by a screening questionnaire at the baseline.

\section{Sensitivity analysis}

This will be carried out for studies with loss to follow-up (LTFU) and studies with high risk of bias on a particular component across studies. We will consider patients loss to follow-up (LTFU) subsequent to randomisation as missing for data analysis and will be explored further for imputation, if it is $>5 \%$. For trials in which the authors report total missing participant data only, without specifying at what stage the participants were missing, we will consider the total sample size and the actual sample size included for final analysis and assume that missing data were equally distributed between the arms. For trials in which the authors reported imputed analysis only, we will use the imputed results for the meta-analysis. We will perform imputation strategies as described by Ebrahim et $a l,{ }^{42}$ and $\mathrm{Akl} e t a l^{43}$ for continuous measures and dichotomous measures, respectively. We will perform this analysis only for the pain relief outcome.

\section{Addressing potential biases}

If there are more than 10 studies for our meta-analysis, funnel plot will be used to assess for publication bias. Trials with low sample size can increase the chances of random error and also show erroneously large treatment effect sizes. Inclusion of such studies in a meta-analysis 
increases the chances of publication bias. ${ }^{44} 45$ As suggested by Moore et $a l^{40}{ }^{40}$ we will consider a sample size threshold of $<50$ to identify a trial as having the potential for publication bias based on low sample size.

\section{Interpretation and reporting}

Reporting of outcomes will be performed as WMD or SMD for continuous outcomes, and relative risks (RR) for dichotomous outcomes, with their 95\% CIs. For dichotomous outcomes, we will also report the findings in measures of absolute risk reduction. Rating of quality of evidence will also be performed using the Grading of Recommendations Assessment, Development and Evaluation (GRADE) approach, by using a 'summary of findings' table.

\section{DISCUSSION}

Treatment of CLBP requires a multimodal approach. Considerations for choosing appropriate medications include a rationale based on underlying mechanism and treatment effectiveness. Since CLBP is recurrent and long standing, it may require long-term treatment involving significant costs to the patient and the payer. Although gabapentinoids are commonly used for the treatment of CLBP, their effectiveness is not clear. Our review will look for existing evidence in the form of RCTs. This will help guide treatment decisions for CLBP, advance patient care based on available evidence, and highlight limitations in existing evidence to direct future research.

\section{Limitations and challenges}

Our review does not include studies that focus primarily on patients with lumbar radicular symptoms. Although there are no existing reviews in this population for the use of gabapentinoids, we felt that addition of such studies will add to the clinical heterogeneity. Lumbar radicular pain has a much pathophysiology and elements leading to neuropathic pain. Since there is a stronger rationale to use gabapentinoids in that population, we feel that results obtained from the inclusion of such studies may potentially lessen the clarity and impact of evidence directed at isolated or predominant CLBP. Despite this exclusion, studies included in our review may still involve considerable heterogeneity. This may be as a result of variability in underlying pathology, duration of chronic pain and presence of other conditions of chronic pain, variability in the time and the method of outcome collection for pain relief or other outcomes. Inclusion of crossover trials in a meta-analysis has its limitations. We have outlined our plan to include and analyse crossover studies in the 'Methods and analysis' section.

\footnotetext{
Author affiliations

${ }^{1}$ Department of Anesthesiology, McMaster University, Michael DeGroote School of Medicine, Hamilton, Ontario, Canada

2Department of Anesthesiology and Biomedical Sciences, Queen's University, Kingston, Ontario, Canada
}

${ }^{3}$ Department of Clinical Epidemiology and Biostatistics, McMaster University, Hamilton, Ontario, Canada

${ }^{4}$ Department of Medicine, McMaster University, Hamilton, Ontario, Canada ${ }^{5}$ Department of Surgery, McMaster University, Hamilton, Ontario, Canada ${ }^{6}$ Department of Neuroanesthesia, National Institute of Mental Health and Neurosciences, Bangalore, India

Acknowledgements The authors acknowledge the assistance provided by Rachel Couban (librarian) in conducting the literature search.

Contributors HS is the primary investigator; conceived the study concept; involved in conduct of the review and analysis and drafted the protocol manuscript. IG is a coinvestigator and content expert on neuropathic pain and analgesic clinical trials and involved in conduct and interpretation of study results. LT is a coinvestigator and involved in methodological supervision and conduct and interpretation of study results. PJD and MB are coinvestigators and involved in methodological supervision and conduct and interpretation of study results. MR, SK and RA are coinvestigators and involved in conduct of the review and manuscript preparation. All authors approve the publication of the protocol.

\section{Competing interests None declared.}

Provenance and peer review Not commissioned; externally peer reviewed.

Open Access This is an Open Access article distributed in accordance with the Creative Commons Attribution Non Commercial (CC BY-NC 4.0) license, which permits others to distribute, remix, adapt, build upon this work noncommercially, and license their derivative works on different terms, provided the original work is properly cited and the use is non-commercial. See: http:// creativecommons.org/licenses/by-nc/4.0/

\section{REFERENCES}

1. Hooten WM, Cohen SP. Evaluation and treatment of low back pain: a clinically focused review for primary care specialists. Mayo Clin Proc 2015;90:1699-718.

2. Juniper M, Le TK, Mladsi D. The epidemiology, economic burden, and pharmacological treatment of chronic low back pain in France, Germany, Italy, Spain and the UK: a literature-based review. Expert Opin Pharmacother 2009;10:2581-92.

3. Parthan A, Evans CJ, Le K. Chronic low back pain: epidemiology, economic burden and patient-reported outcomes in the USA. Expert Rev Pharmacoecon Outcomes Res 2006;6:359-69.

4. Hoy D, Bain C, Williams G, et al. A systematic review of the global prevalence of low back pain. Arthritis Rheum 2012;64:2028-37.

5. Murray CJ, Atkinson C, Bhalla $\mathrm{K}$, et al. The state of US health 1990-2010: burden of diseases, injuries, and risk factors. JAMA 2013;310:591-608.

6. Machado LA, Kamper SJ, Herbert RD, et al. Analgesic effects of treatments for non-specific low back pain: a meta-analysis of placebo-controlled randomized trials. Rheumatology (Oxf) 2009:48:520-7.

7. Rathmell JP. A 50 -year-old man with chronic low back pain. JAMA 2008;299:2066-77.

8. Carragee EJ. Clinical practice. Persistent low back pain. $N$ Engl J Med 2005;352:1891-8.

9. Deyo RA, Weinstein JN. Low back pain. $N$ Engl J Med 2001;344:363-70.

10. Kaki AM, El-Yaski AZ, Youseif E. Identifying neuropathic pain among patients with chronic low-back pain: use of the Leeds Assessment of Neuropathic Symptoms and Signs pain scale. Reg Anesth Pain Med 2005;30:422-8.

11. Nicholson B. Differential diagnosis: nociceptive and neuropathic pain. Am J Manag Care 2006;12(9 Suppl):S256-62.

12. Smart $\mathrm{KM}$, Blake $\mathrm{C}$, Staines $\mathrm{A}$, et al Clinical indicators of 'nociceptive', 'peripheral neuropathic' and 'central' mechanisms of musculoskeletal pain. A Delphi survey of expert clinicians. Man Ther 2010;15:80-7.

13. Latremoliere A, Woolf CJ. Central sensitization: a generator of pain hypersensitivity by central neural plasticity. J Pain 2009;10:895-926.

14. Nijs J, Apeldoorn A, Hallegraeff $\mathrm{H}$, et al. Low back pain: guidelines for the clinical classification of predominant neuropathic, nociceptive, or central sensitization pain. Pain Physician 2015;18:E333-46. 
15. Roussel NA, Nijs J, Meeus M, et al. Central sensitization and altered central pain processing in chronic low back pain: fact or myth? Clin J Pain 2013;29:625-38.

16. Smart KM, Blake C, Staines A, et al. Mechanisms-based classifications of musculoskeletal pain: part 1 of 3 : symptoms and signs of central sensitisation in patients with low back $(+/-$ leg) pain. Man Ther 2012;17:336-44.

17. Smith $\mathrm{BH}$, Torrance $\mathrm{N}$, Bennett MI, et al. Health and quality of life associated with chronic pain of predominantly neuropathic origin in the community. Clin J Pain 2007;23:143-9.

18. Baron R, Binder A, Wasner G. Neuropathic pain: diagnosis, pathophysiological mechanisms, and treatment. Lancet Neurol 2010;9:807-19.

19. Haanpaa M, Attal N, Backonja M, et al. NeuPSIG guidelines on neuropathic pain assessment. Pain 2011:152:14-27.

20. La Cesa S, Tamburin S, Tugnoli V, et al. How to diagnose neuropathic pain? The contribution from clinical examination, pain questionnaires and diagnostic tests. Neurol Sci 2015;36:2169-75

21. Morlion B. The relevance of neuropathic components in chronic back pain. Curr Med Res Opin 2011;27:2067-8.

22. Berger A, Dukes EM, Oster G. Clinical characteristics and economic costs of patients with painful neuropathic disorders. J Pain 2004:5:143-9.

23. White AP, Arnold PM, Norvell DC, et al. Pharmacologic management of chronic low back pain: synthesis of the evidence. Spine 2011;36(21 Suppl):S131-43.

24. Gajraj NM. Pregabalin: its pharmacology and use in pain management. Anesth Analg 2007;105:1805-15.

25. Bennett MI, Simpson KH. Gabapentin in the treatment of neuropathic pain. Palliat Med 2004;18:5-11.

26. Finnerup NB, Attal N, Haroutounian S, et al. Pharmacotherapy for neuropathic pain in adults: a systematic review and meta-analysis Lancet Neurol 2015;14:162-73.

27. Morlion B. Pharmacotherapy of low back pain: targeting nociceptive and neuropathic pain components. Curr Med Res Opin 2011;27:11-33.

28. Patel R, Dickenson AH. Mechanisms of the gabapentinoids and alpha 2 delta- 1 calcium channel subunit in neuropathic pain. Pharmacol Res Perspect 2016;4:e00205.

29. Sætterstrøm B, Poulsen PB, Olsen J, et al. PSY9 real-life cost-analyses of chronic low-back pain patients with neuropathic pain components in Denmark. Value Health 2012;15:A509.

30. Maizels M, McCarberg B. Antidepressants and antiepileptic drugs for chronic non-cancer pain. Am Fam Physician 2005;71:483-90.
31. Toth C. Drug safety evaluation of pregabalin. Expert Opin Drug Saf 2012;11:487-502.

32. Salerno SM, Browning R, Jackson JL. The effect of antidepressant treatment on chronic back pain: a meta-analysis. Arch Intern Med 2002;162:19-24.

33. Chou R, Huffman LH. Medications for acute and chronic low back pain: a review of the evidence for an American Pain Society/ American College of Physicians clinical practice guideline. Ann Intern Med 2007;147:505-14.

34. Romano CL, Romano D, Lacerenza M. Antineuropathic and antinociceptive drugs combination in patients with chronic low back pain: a systematic review. Pain Res Treat 2012;2012:154781.

35. Dworkin RH, Turk DC, Farrar JT, et al. Core outcome measures for chronic pain clinical trials: IMMPACT recommendations. Pain 2005;113:9-19.

36. Shamseer L, Moher D, Clarke M, et al. Preferred reporting items for systematic review and meta-analysis protocols (PRISMA-P) 2015: elaboration and explanation. BMJ 2015;349:g7647.

37. Viera AJ, Garrett JM. Understanding interobserver agreement: the kappa statistic. Fam Med 2005;37:360-3.

38. Higgins JPT, Green S. Cochrane handbook for systematic reviews of interventions, Version 5.1.0. The Cochrane Collaboration, 2011 http://www.cochrane-handbook.org

39. Chan AW, Altman DG. Identifying outcome reporting bias in randomised trials on PubMed: review of publications and survey of authors. BMJ 2005;330:753.

40. Andrew Moore R, Eccleston C, Derry S, et al. "Evidence" in chronic pain-establishing best practice in the reporting of systematic reviews. Pain 2010;150:386-9.

41. Moore RA, Gavaghan D, Tramer MR, et al. Size is everything-large amounts of information are needed to overcome random effects in estimating direction and magnitude of treatment effects. Pain 1998;78:209-16.

42. Ebrahim S, Johnston BC, Akl EA, et al. Addressing continuous data measured with different instruments for participants excluded from trial analysis: a guide for systematic reviewers. J Clin Epidemiol 2014:67:560-70.

43. Akl EA, Johnston BC, Alonso-Coello $P$, et al. Addressing dichotomous data for participants excluded from trial analysis: a guide for systematic reviewers. PLoS One 2013;8:e57132.

44. Ioannidis JP, Cappelleri JC, Lau J. Issues in comparisons between meta-analyses and large trials. JAMA 1998;279:1089-93.

45. Sterne JA, Gavaghan D, Egger M. Publication and related bias in meta-analysis: power of statistical tests and prevalence in the literature. J Clin Epidemiol 2000;53:1119-29. 